\title{
DERMATOLOGIC HISTORY
}

\section{Norman Orentreich: Father of Modern Hair Transplantation}

Michael Phan $\mathrm{BS}^{1}$, Jonathan Phan $\mathrm{MS}^{1}$

${ }^{1}$ University of Texas Medical Branch, School of Medicine, Galveston TX

Norman Orentreich, MD is universally regarded as the father of modern hair transplantation. He was born in New York City on December 26, 1922. He completed his undergraduate studies at the College of New York and served in the US Navy Medical Corps from 1943-1945. Dr. Orentriech attended medical school at the New York University of College of Medicine and received his dermatology training at NYU's Skin and Cancer Unit. ${ }^{1}$

While studying the pathophysiology of diverse dermatologic conditions using hairbearing punch grafts in the early 1950s, he observed that autografts from hair-bearing areas of the scalp continued to grow hair when implanted in areas of physiological male-pattern alopecia. ${ }^{2}$ This observation established the principle of donor dominance: transplanted autografts maintained their integrity and characteristics independent of the recipient site. After successfully transplanting hair in 65 patients, he published his results in $1959 .{ }^{2}$ Dr. Orentreich understood the limitations of his technique and sought methods to improve his patient's cosmetic outcomes. By 1985 , he created minigrafts containing three to five hairs and overlapped his grafts to address patchy hair growth. Dr. Orentreich also incorporated the use of intralesional corticosteroid to prevent hypertrophic and keloidal scarring and dermabrasion to treat elevated grafts. $^{3}$ His follicle-preserving extraction technique paved way for the development of follicular unit transplantation and extraction. ${ }^{1}$

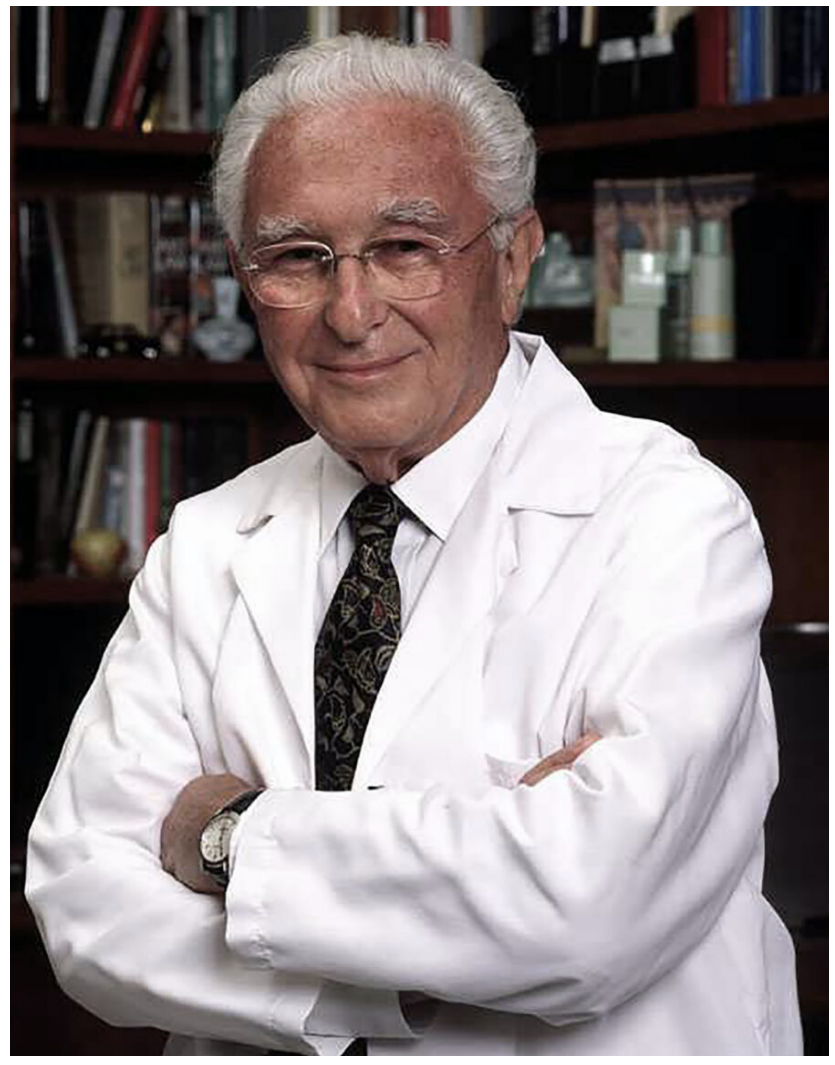

Dr. Orentreich's interests extend beyond hair transplantation. He founded the Orentreich Foundation for the Advancement of Science for aging and nutrition research in 1961. In 1968, the Lauder Family recruited Dr. Orentreich and Vogue editor September 2020 Volume 4 Issue 5 
Carol Phillips to create Clinique, the world's first allergy-tested, fragrance-free, dermatologist-guided cosmetic line. He was also elected the first president of the American Society for Dermatologic Surgery in $1970 .^{1}$

Dr. Orentreich established the current field of hair transplantation with his visionary discoveries. His contributions to dermatology have benefited many people with hair loss in the $20^{\text {th }}$ century.

Conflict of Interest Disclosures: None

Funding: None

Corresponding Author:

Michael Phan

1005 Harborside Dr F5

Galveston, TX 77555

Email: miphan@utmb.edu

\section{References:}

1. Refs Orentreich Medical Group. Our Founder. http://orentreich.com/about-us/our-founder/. Accessed July 6, 2020.

2. Orentreich N. Autografts in alopecias and other selected dermatological conditions. Ann NY Acad Sci. 1959;83:463-479. doi:10.1111/j.17496632.1960.tb40920.x

3. Orentreich DS, Orentreich N. Hair transplantation. J Dermatol Surg Oncol. 1985;11(3):319-324. doi:10.1111/j.15244725.1985.tb03010.x 